\title{
Dynamics in a Nonautonomous Nicholson-Type Delay System
}

\author{
Ahmadjan Muhammadhaji (iD) and Azhar Halik \\ College of Mathematics and System Sciences, Xinjiang University, Urumqi 830046, China \\ Correspondence should be addressed to Ahmadjan Muhammadhaji; ahmatjanam@aliyun.com
}

Received 16 November 2020; Revised 6 April 2021; Accepted 30 April 2021; Published 11 May 2021

Academic Editor: Antonio Di Crescenzo

Copyright (C) 2021 Ahmadjan Muhammadhaji and Azhar Halik. This is an open access article distributed under the Creative Commons Attribution License, which permits unrestricted use, distribution, and reproduction in any medium, provided the original work is properly cited.

A kind of Nicholson-type delay system is considered. Several conditions on the ultimate boundedness, extinction, permanence, periodic solution, and global attractivity of the system are established by employing the inequality techniques and comparison method and constructing suitable Lyapunov functional.

\section{Introduction}

In order to describe the population of the Australian sheep blowfly and based on the experimental data of Nicholson $[1,2]$, Gurney et al. [3] first proposed the nonlinear autonomous delay equation:

$$
\dot{z}(t)=-\alpha z(t)+q z(t-\theta) e^{-\beta z(t-\theta)}, \quad \alpha, q, \beta, \theta \in(0, \infty) .
$$

In system (1), $z(t)$ is the population size, $q$ is the maximum per capita daily egg production, $1 / \beta$ is the size at which the population reproduces at its maximum rate, $\alpha$ is the per capita daily adult death rate, and $\theta$ is the generation time.

It is well known that system (1) not only has profound practical significance but also will enrich and perfect the models on the Nicholson blowflies to some extent. From the published papers [4-16], we can also find several other similar interesting models on the Nicholson blowflies. For example, Saker and Agarwal [4] studied the periodic solution of the following nonautonomous periodic Nicholson's blowfly system:

$$
\dot{z}(t)=-\alpha(t) z(t)+q(t) z(t-m \omega) e^{-\beta z(t-\omega)},
$$

and derived several sufficient conditions for the global attractivity of the periodic solution, where $\beta$ is a positive scalar, $m$ is a positive integer, and $\alpha(t)>0, q(t)>0$ are periodic functions with period $\omega$. In [5], the authors considered the following autonomous Nicholson-type delay systems:

$$
\begin{aligned}
& \dot{z}_{1}(t)=-a_{1} z_{1}(t)+b_{1} z_{2}(t)+c_{1} z_{1}(t-\gamma) e^{-z_{1}(t-\gamma)} \\
& \dot{z}_{2}(t)=-a_{2} z_{2}(t)+b_{2} z_{1}(t)+c_{2} z_{2}(t-\gamma) e^{-x_{2}(t-\gamma)}
\end{aligned}
$$

and obtained some sufficient conditions on the existence of positive global solutions, lower and upper estimations of solutions, and the existence and uniqueness of a positive equilibrium, where $a_{1}, a_{2}, b_{1}, b_{2}, c_{1}, c_{2}$, and $\gamma$ are nonnegative constants.

In the real world, the habitat environment of the population will change along with time, and this leads to changes in the growth characteristics of these populations. However, the autonomous system irrespective of the environmental changes has some limitations in mathematical modeling of ecological systems. Therefore, we should introduce nonautonomous case into our study on the dynamical behaviors of Nicholson's blowflies, and it is also valuable and important to study Nicholson's blowfly populations in a nonautonomous environment. Based on the above models and analysis, it is necessary to study model (3) which contains the nonautonomous case. Hence, we consider the following nonautonomous Nicholson-type delay system:

$$
\begin{aligned}
& \dot{z}_{1}(t)=-a_{1}(t) z_{1}(t)+b_{1}(t) z_{2}(t)+c_{1}(t) z_{1}(t-\gamma) e^{-z_{1}(t-\gamma)}, \\
& \dot{z}_{2}(t)=-a_{2}(t) z_{2}(t)+b_{2}(t) z_{1}(t)+c_{2}(t) z_{2}(t-\gamma) e^{-z_{2}(t-\gamma)},
\end{aligned}
$$

where $z_{i}(t)(i=1,2)$ is the size of the population at time $t$, $a_{i}(t)(i=1,2)$ is the per capita daily adult death rate at time $t$, 
$c_{i}(t)(i=1,2)$ is the maximum per capita daily egg production at time $t, b_{i}(t)(i=1,2)$ represent the dispersal (transition) rates at time $t$, and $\gamma$ is the generation time.

As far as we know, the main foci of theoretical studies in biological and ecological dynamical systems are the permanence, extinction of the populations, periodic solution, and global attractivity of the system. Hence, in the present paper, our main aim is to study the aforementioned dynamical behaviors of system (4).

The organization of this paper is as follows. In the next section, we will present some basic assumptions, definitions, and main lemmas. In Section 3, conditions for the permanence, extinction, and periodic solution of the system are considered. In Section 4, we establish conditions for global attractivity of the system. In Section 5, a numerical example is given to illustrate that our main results are applicable. Conclusions are finally drawn in Section 6.

\section{Preliminaries}

The following are the basic assumptions which system (4) satisfies.

$\left(H_{1}\right) \gamma>0$ is a scalar, and $a_{i}(t), b_{i}(t), c_{i}(t)(i=1,2)$ are positive, bounded, and continuous functions on $[0,+\infty)$.

$\left(H_{2}\right) \gamma>0$ is a scalar, and $a_{i}(t), b_{i}(t), c_{i}(t)(i=1,2)$ are positive $\omega$-periodic continuous functions on $[0, \omega]$.

The following is the initial condition of system (4):

$$
\begin{aligned}
& z_{1}(t)=\zeta_{1}(t), \\
& z_{2}(t)=\zeta_{2}(t), \quad \forall t \in[-\gamma, 0],
\end{aligned}
$$

where $\zeta_{1}(t), \zeta_{2}(t)$ are continuous and nonnegative functions defined on $[-\gamma, 0)$ satisfying $\zeta_{1}(0)>0, \zeta_{2}(0)>0$.

Let $H(t)$ be any continuous, bounded function defined on $[0, \infty)$, and we set $H^{L}=\inf _{t \in[0, \infty)}\{H(t)\}$ and $H^{M}=$ $\sup _{t \in[0, \infty)}\{H(t)\}$.

Now, we present some useful definitions and lemmas.

Definition 1. System (4) is said to be permanent if there exist positive constants $m, M$, and $T_{0}$, such that each positive solution $\left(z_{1}(t), z_{2}(t)\right)$ of system (4) with any positive initial value $\varphi$ fulfills $m \leq z_{i}(t) \leq M(i=1,2)$ for all $t \geq T_{0}$, where $T_{0}$ may depend on $\varphi$.

Definition 2. System (4) is said to be extinct if any positive solution $\left(z_{1}(t), z_{2}(t)\right)$ of system (4) with any positive initial value $\varphi$ fulfills

$$
\lim _{t \longrightarrow+\infty} z_{i}(t)=0, \quad(i=1,2)
$$

Definition 3. System (4) is said to be global attractive, if for any two positive solutions $\left(z_{1}(t), z_{2}(t)\right.$ and $\left(y_{1}(t), y_{2}(t)\right)$ of system (4), one has

$$
\lim _{t \longrightarrow+\infty}\left(x_{i}(t)-y_{i}(t)\right)=0, \quad i=1,2 .
$$

Lemma 1 (see [17]). If $\dot{y}(t) \geq(\leq) B-A y(t)$ and $A>0$, $B>0$, then we have

$$
y(t) \geq(\leq) \frac{B}{A}\left[1+\left(\frac{A y(0)}{B}-1\right) e^{-A t}\right],
$$

where $t \geq 0$ and $y(0)>0$.

Lemma 2 (see [17]). If $A>0, B>0, C>0$, and $\sigma>0$, then for delay system $\dot{y}(t)=A y(t-\sigma)-B y(t)-C y^{2}(t)$, when $y(t)>0$ and $t \in[-\sigma, 0]$, we have

(1) If $A>B$, then $\lim _{t \longrightarrow+\infty} y(t)=(A-B) / C$.

(2) If $A<B$, then $\lim _{t \longrightarrow+\infty} y(t)=0$.

Lemma 3 (see [17]). If $A>0, B>0$, and $\sigma>0$, then for delay system $\dot{y}(t)=A y(t-\sigma)-B y(t)$, when $y(t)>0$ and $t \in[-\sigma, 0]$, we have

(1) If $A<B$, then $\lim _{t \rightarrow+\infty} y(t)=0$.

(2) If $A>B$, then $\lim _{t \rightarrow+\infty} y(t)=+\infty$.

Lemma 4 (Barbalat's theorem). Let $f$ be a nonnegative function defined on $[0, \infty)$, such that $f$ is integrable on $[0, \infty)$ and uniformly continuous on $[0, \infty)$. Then, $\lim _{t \rightarrow \infty} f(t)=0$.

\section{Positivity, Permanence, Extinction, and Periodic Solution}

Theorem 1. The solutions of system (4) with initial conditions (5) are positive for all $t \geq 0$.

Proof. Let $\left(z_{1}(t), z_{2}(t)\right)$ be a solution of system (4) with initial conditions (5). First, it follows from the first equation of system (4) for $t \in[0, \gamma]$ that

$$
\begin{aligned}
\dot{z}_{1}(t) & =-a_{1}(t) z_{1}(t)+b_{1}(t) z_{2}(t)+c_{1}(t) \zeta_{1}(t-\gamma) e^{-\zeta_{1}(t-\gamma)} \\
& \geq-a_{1}(t) z_{1}(t)
\end{aligned}
$$

since $\zeta_{1}(t)$ is a nonnegative continuous function defined on $t \in[-\gamma, 0]$. Therefore, a standard comparison argument shows that

$$
z_{1}(t) \geq z_{1}(0) e^{-\int_{0}^{t} a_{1}(s) \mathrm{d} s} .
$$

Thus, $z_{1}(t)>0$ for $t \in[0, \gamma]$.

Next, by the second equation of system (4), for $t \in[0, \gamma]$, we directly obtain

$$
z_{2}(t) \geq z_{2}(0) e^{-\int_{0}^{t} a_{2}(s) \mathrm{d} s}>0 .
$$


In a similar way, we treat the intervals $[\gamma, 2 \gamma], \ldots,[n \gamma,(n+1) \gamma], n \in N$. Thus, $z_{1}(t)>0, z_{2}(t)>0$ for all $t>0$. This completes the proof.

Now, we will introduce a useful inequality. Since the function $(1-x) / e^{x}$ is decreasing with the range $[0,1]$, it follows easily that there exists a unique $k \in(0,1)$ such that

$$
\frac{1-k}{e^{k}}=\frac{1}{e^{2}}
$$

Obviously,

$$
\sup _{x \geq k}\left|\frac{1-x}{e^{x}}\right|=\frac{1}{e^{2}}
$$

Moreover, since $x e^{-x}$ increases on $[0,1]$ and decreases on $[1, \infty)$, let $\tilde{k}$ be the unique number in $(1, \infty)$ such that

$$
k e^{-k}=\tilde{k} e^{-\tilde{k}}
$$

Hence,

$$
\max _{x \geq 0} x e^{-x} \leq \frac{1}{e}
$$

Also, it is not difficult to find the numbers $k$ and $\widetilde{k}$ by calculation, where $k \approx 0.72153545723892615 \in(0,1)$ and $\widetilde{k} \approx 1.342275592250851 \in(1,+\infty)$. Inequality $(15)$ can be found in [5-11].

Theorem 2. Assume that $\left(H_{1}\right)$ holds and $A_{1}>0, A_{2}>0$; then, the solutions of system (4) are ultimately bounded from above, where $A_{1}=a_{1}^{L}-b_{2}^{M}, A_{2}=a_{2}^{L}-b_{1}^{M}$.

Proof. Let $\left(z_{1}(t), z_{2}(t)\right)$ be any positive solution of system (4). Define the function

$$
W(t)=z_{1}(t)+z_{2}(t) .
$$

Computing the derivative of $W(t)$ and by (15), we get

$$
\begin{gathered}
\dot{W}(t)=\dot{z}_{1}(t)+\dot{z}_{2}(t) \leq-A_{1} z_{1}(t)-A_{2} z_{2}(t) \\
+\left(c_{1}^{M}+c_{2}^{M}\right) \frac{1}{e} \leq B_{1}-B_{2} W(t),
\end{gathered}
$$

where $B_{1}=\left(c_{1}^{M}+c_{2}^{M}\right)(1 / e)$ and $B_{2}=\min \left\{A_{1}, A_{2}\right\}$. Then, by Lemma 1, we have

$$
W(t) \leq \frac{B_{1}}{B_{2}}+\left(W(0)-\frac{B_{1}}{B_{2}}\right) e^{-B_{2} t},
$$

which yields

$$
\lim _{t \longrightarrow+\infty} W(t)=\lim _{t \longrightarrow+\infty}\left(z_{1}(t)+z_{2}(t)\right) \leq \frac{B_{1}}{B_{2}} .
$$

This implies that any positive solution of system (4) is ultimately bounded. Thus, there exist positive constants $T_{0}$ and $M$ such that $z_{i}(t)<M(i=1,2)$ for $t>T_{0}$, where $M=B_{1} / B_{2}$.
Corollary 1. If $A_{1}^{\prime}>0, A_{2}^{\prime}>0$, then the solutions of system (3) are ultimately bounded from above, where $A_{1}^{\prime}=a_{1}-b_{2}$, $A_{2}^{\prime}=a_{2}-b_{1}$.

Theorem 3. System (4) is permanent if the conditions of Theorem 1 hold and $\left(c_{i}^{L} / e^{M}\right)-a_{i}^{M}>0(i=1,2)$.

Proof. Assume that $\left(z_{1}(t), z_{2}(t)\right)$ is any positive solution of system (4). Firstly, by Theorem 1 , there exist positive constants $T_{0}$ and $M$ such that $z_{i}(t)<M(i=1,2)$ for $t>T_{0}$. Next, from system (4), for $t>T_{0}$, we get

$$
\dot{z}_{1}(t) \geq \frac{c_{1}^{L}}{e^{M}} z_{1}(t-\gamma)-a_{1}^{M} z_{1}(t)-z_{1}^{2}(t) .
$$

Note the following equation:

$$
\dot{w}(t)=\frac{c_{1}^{L}}{e^{M}} w(t-\gamma)-a_{1}^{M} w(t)-w^{2}(t) .
$$

From Lemma 2, we derive

$$
\lim _{t \longrightarrow+\infty} w(t)=\frac{c_{1}^{L}}{e^{M}}-a_{1}^{M} \triangleq m_{1} .
$$

By comparison, there exists a $T_{1}>T_{0}$ such that $z_{1}(t) \geq m_{1}$ for $t \geq T_{1}$. Finally, from system (4), for $t>T_{0}$, we have

$$
\dot{z}_{2}(t) \geq \frac{c_{2}^{L}}{e^{M}} z_{2}(t-\gamma)-a_{2}^{M} z_{2}(t)-z_{2}^{2}(t) .
$$

Similar to the above discussion, we have

$$
\lim _{t \rightarrow+\infty} z_{2}(t) \geq \frac{c_{1}^{L}}{e^{M}}-a_{1}^{M} \triangleq m_{2} .
$$

Then, there exists a $T_{2}>T_{0}$ such that $z_{2}(t) \geq m_{2}$ for $t \geq T_{2}$.

Corollary 2. System (3) is permanent if the conditions of Corollary 1 hold and $\left(c_{i} / e^{M}\right)-a_{i}>0(i=1,2)$.

Theorem 4. System (4) is extinct if $\left(H_{1}\right)$ holds and $A>C$, where $A=\min \left\{a_{1}^{L}-b_{2}^{M}, a_{2}^{L}-b_{1}^{M}\right\}$ and $C=\max \left\{c_{1}^{M}, c_{2}^{M}\right\}$.

Proof. Let $\left(z_{1}(t), z_{2}(t)\right)$ be any positive solution of system (4). Define the function

$$
Z(t)=z_{1}(t)+z_{2}(t)
$$

Computing the derivative of $Z(t)$, we get

$$
\begin{aligned}
\dot{Z}(t)= & \dot{z}_{1}(t)+\dot{z}_{2}(t) \leq-A_{1} z_{1}(t)-A_{2} z_{2}(t) \\
& +c_{1}^{M} z_{1}(t-\gamma)+c_{2}^{M} z_{2}(t-\gamma) \leq C Z(t-\gamma)-A Z(t),
\end{aligned}
$$

where $C=\max \left\{c_{1}^{M}, c_{2}^{M}\right\}$ and $A=\min \left\{A_{1}, A_{2}\right\}$.

Note the following equation:

$$
\dot{H}(t)=C H(t-\gamma)-A H(t) .
$$

Then, from Lemma 3, we get 


$$
\lim _{t \rightarrow+\infty} H(t)=0
$$

Hence, there exists a $T_{3}>0$ such that $Z(t)=z_{1}$ $(t)+z_{2}(t) \longrightarrow 0$, which yields $z_{i}(t) \longrightarrow 0(i=1,2)$ for $t>T_{3}$.

Corollary 3. System (3) is extinct if $A^{\prime}>C^{\prime}$, where $A^{\prime}=$ $\min \left\{a_{1}-b_{2}, a_{2}-b_{1}\right\}$ and $C^{\prime}=\max \left\{c_{1}, c_{2}\right\}$.

Applying Lemma 4 in [18] and from Theorem 2, we get the following.

Corollary 4. Assume that $\left(H_{2}\right)$ holds and $A_{i}>0,\left(c_{i}^{L} /\right.$ $\left.e^{M}\right)-a_{i}^{M}>0(i=1,2)$; then, system (4) is permanent and has at least one positive $\omega$-periodic solution, where $A_{i}(i=1,2)$ are given in Theorem 1.

\section{Global Attractivity}

Theorem 5. If $\left(H_{1}\right)$ holds and $D_{i}>0(i=1,2)$, then system (4) is globally attractive, where

$$
\begin{aligned}
& \lim \inf _{t \rightarrow+\infty}\left[a_{1}(t)-b_{2}(t)-\frac{c_{1}(t+\gamma)}{e^{2}}\right]=: D_{1}, \\
& \lim \inf _{t \rightarrow+\infty}\left[a_{2}(t)-b_{1}(t)-\frac{c_{2}(t+\gamma)}{e^{2}}\right]=: D_{2} .
\end{aligned}
$$

Proof. Suppose that $\left(z_{1}(t), z_{2}(t)\right)$ and $\left(y_{1}(t), y_{2}(t)\right)$ are any two positive solutions of system (4); then, we define a Lyapunov functional as follows:

$$
V(t)=\sum_{i=1}^{2}\left(\left|z_{i}(t)-y_{i}(t)\right|+\int_{t-\gamma}^{t} c_{i}(s+\gamma)\left|z_{i}(s) e^{-z_{i}(s)}-y_{i}(s) e^{-y_{i}(s)}\right| \mathrm{d} s\right)
$$

Computing the Dini derivative of Lyapunov functional $V(t)$, we get

$$
\begin{aligned}
D^{+} V(t)= & \operatorname{sign}\left(z_{1}(t)-y_{1}(t)\right)\left[\begin{array}{l}
-a_{1}(t)\left(z_{1}(t)-y_{1}(t)\right)+b_{1}(t)\left(z_{2}(t)-y_{2}(t)\right) \\
z_{1}(t-\gamma) e^{-z_{1}(t-\gamma)}-y_{1}(t-\gamma) e^{-y_{1}(t-\gamma)}+c_{1}(t)
\end{array}\right] \\
& +\operatorname{sign}\left(z_{2}(t)-y_{2}(t)\right)\left[\begin{array}{c}
-a_{2}(t)\left(z_{2}(t)-y_{2}(t)\right)+b_{2}(t)\left(z_{1}(t)-y_{1}(t)\right) \\
+c_{2}(t)\left(z_{2}(t-\gamma) e^{-z_{2}(t-\gamma)}-y_{2}(t-\gamma) e^{-y_{2}(t-\gamma)}\right)
\end{array}\right] \\
& +\sum_{i=1}^{2} c_{i}(t+\gamma)\left|z_{i}(t) e^{-z_{i}(t)}-y_{i}(t) e^{-y_{i}(t)}\right|-\sum_{i=1}^{2} c_{i}(t)\left|z_{i}(t-\gamma) e^{-z_{i}(t-\gamma)}-y_{i}(t-\gamma) e^{-y_{i}(t-\gamma)}\right| \\
\leq & -\left(a_{1}(t)-b_{2}(t)\right)\left|z_{1}(t)-y_{1}(t)\right|-\left(a_{2}(t)-b_{1}(t)\right)\left|z_{2}(t)-y_{2}(t)\right|+\sum_{i=1}^{2} c_{i}(t+\gamma)\left|z_{i}(t) e^{-z_{i}(t)}-y_{i}(t) e^{-y_{i}(t)}\right| .
\end{aligned}
$$

Note that

$\left|p e^{-p}-q e^{-q}\right|=\left|\frac{1-(p+\theta(q-p))}{e^{p+\theta(q-p)}}\right||p-q| \leq \frac{1}{e^{2}}|p-q|$, where $p, q \in[k,+\infty), 0<\theta<1$.

From (29) and (30), we have 


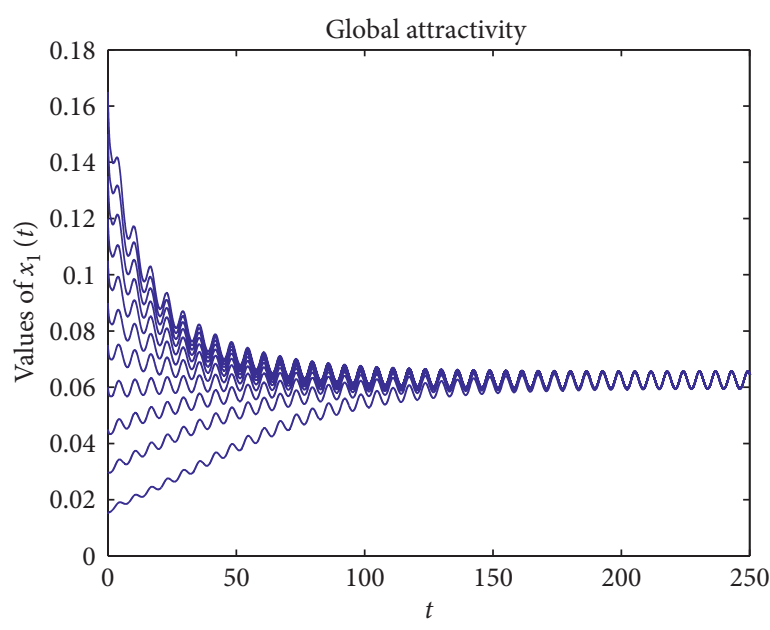

(a)

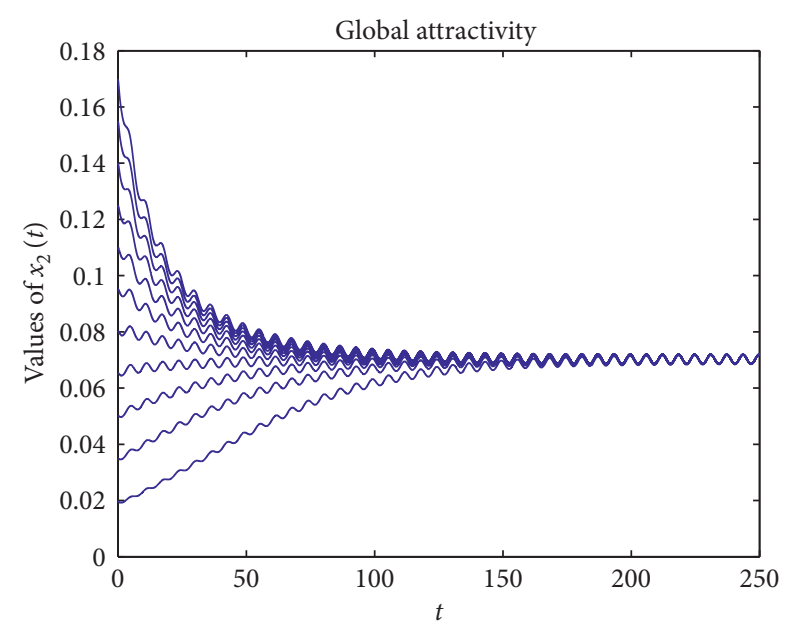

(b)

Figure 1: Global attractivity of system (39). Here, we take different initial values.

$$
\begin{aligned}
& D^{+} V(t)-\left(a_{1}(t)-b_{2}(t)\right)\left|z_{1}(t)-y_{1}(t)\right|-\left(a_{2}(t)-b_{1}(t)\right)\left|z_{2}(t)-y_{2}(t)\right| \leq+\frac{c_{1}(t+\gamma)}{e^{2}}\left|z_{1}(t)-y_{1}(t)\right|+\frac{c_{2}(t+\gamma)}{e^{2}}\left|z_{2}(t)-y_{2}(t)\right| \\
& =-\left(a_{1}(t)-b_{2}(t)-\frac{c_{1}(t+\gamma)}{e^{2}}\right)\left|z_{1}(t)-y_{1}(t)\right|-\left(a_{2}(t)-b_{1}(t)-\frac{c_{2}(t+\gamma)}{e^{2}}\right)\left|z_{2}(t)-y_{2}(t)\right| \\
& \leq-D_{1}\left|z_{1}(t)-y_{1}(t)\right|-D_{2}\left|z_{1}(t)-y_{1}(t)\right| \\
& \leq-\sum_{i=1}^{2} D\left|z_{i}(t)-y_{i}(t)\right| .
\end{aligned}
$$

Integrating from 0 to $t$ on both sides of (33) produces

$$
\begin{array}{r}
V(t)+D \int_{0}^{t}\left(\sum_{i=1}^{2}\left|z_{i}(s)-y_{i}(s)\right|\right) \mathrm{d} s \leq V(0), \\
V(t)+\sum_{i=1}^{2} D\left|x_{i}(t)-y_{i}(t)\right| \leq V(0) .
\end{array}
$$

Hence, we have

$$
\sum_{i=1}^{2}\left|x_{i}(t)-y_{i}(t)\right|<\frac{V(0)}{D}
$$

From boundedness of the solutions, for $(i=1,2)$, we can get that $\left|z_{i}(t)-y_{i}(t)\right|$ and $\left(\dot{z}_{i}(t)-\dot{y}_{i}(t)\right)$ remain bounded on $[0, \infty)$. Hence, $\left|z_{i}(t)-y_{i}(t)\right|$ is uniformly continuous on $[0, \infty)$. From Barbalat's theorem, for $(i=1,2)$, we can get that

$$
\lim _{t \rightarrow \infty}\left|z_{i}(t)-y_{i}(t)\right|=0
$$

Hence, for $(i=1,2)$, we get

$$
\lim _{t \longrightarrow \infty}\left(z_{i}(t)-y_{i}(t)\right)=0 .
$$

Corollary 5. If $D_{i}^{\prime}>0(i=1,2)$, then system (3) is globally attractive, where

$$
\begin{aligned}
& a_{1}-b_{2}-\frac{c_{1}}{e^{2}}=: D_{1}^{\prime}, \\
& a_{2}-b_{1}-\frac{c_{2}}{e^{2}}=: D_{2}^{\prime} .
\end{aligned}
$$

Corollary 6. System (4) has a globally attractive positive $\omega-$ periodic solution if the conditions of Corollary 4 hold and $D_{i}>0(i=1,2)$, where $D_{i}(i=1,2)$ are given in Theorem 4. 


\section{One Example}

Example 1. We consider the following system:

$$
\begin{aligned}
& \dot{z}_{1}(t)=-(1.6+0.35 \cos (t)) z_{1}(t)+\left(0.75+0.1 \cos (t) z_{2}(t)+0.8+0.15 \cos (t)\right) z_{1}(t-1) e^{-z_{1}(t-1)} \\
& \dot{z}_{2}(t)=-(1.55+0.25 \cos (t)) z_{2}(t)+(0.8+0.15 \cos (t)) z_{1}(t)+(0.9+0.1 \cos (t)) z_{2}(t-1) e^{-z_{2}(t-1)}
\end{aligned}
$$

Directly from calculation, we get

$$
\begin{aligned}
& D_{1} \approx 0.2614, \\
& D_{2} \approx 0.2147 .
\end{aligned}
$$

The global attractivity of system (39) is shown in Figure 1.

\section{Conclusion}

Throughout the paper, we investigate a kind of nonautonomous Nicholson-type delay system. Firstly, based on the inequality techniques and comparison method, we derived several conditions on the boundedness, permanence, extinction, and positive periodic solution. Secondly, the conditions on the global attractivity of the system were derived by employing the Lyapunov function method. Meanwhile, as an application of the results in this paper, we also study autonomous system (3) and obtain several conditions on the aforementioned dynamical behaviors of system (3). We have an interesting topic, such as the study on the ultimate boundedness, extinction, permanence, periodic solution, and global attractivity of the following nonautonomous Nicholson-type delay system:

$$
\dot{z}_{i}(t)=-a_{i}(t) z_{i}(t)+\sum_{j=1, j \neq i}^{n} b_{i j}(t) z_{j}(t)+c_{j}(t) z_{j}(t-\gamma(t)) e^{-z_{i}(t-\gamma(t))}, \quad i=1,2, \ldots, n .
$$

We deserve these abovementioned topics for a future investigation.

\section{Data Availability}

No data were used to support this study.

\section{Conflicts of Interest}

The authors declare that they have no conflicts of interest.

\section{Acknowledgments}

This study was supported by the National Natural Science Foundation of Xinjiang (grant no. 2021D01C067) and the National Natural Science Foundation of China (grant nos. 11662020 and 11861063).

\section{References}

[1] A. J. Nicholson, "Competition for food amongst Lucilia Cuprina larvae," in Proceedings of the 8th International Congress of Entomology, vol. 15, pp. 277-281, Stockholm, Sweden, 1948.

[2] A. Nicholson, "An outline of the dynamics of animal populations," Australian Journal of Zoology, vol. 2, no. 1, pp. 9-65, 1954.

[3] W. S. C. Gurney, S. P. Blythe, and R. M. Nisbet, "Nicholson's blowflies revisited," Nature, vol. 287, no. 5777, pp. 17-21, 1980.
[4] S. H. Saker and S. Agarwal, "Oscillation and global attractivity in a periodic Nicholson's blowflies model," Mathematical and Computer Modelling, vol. 35, no. 7-8, pp. 719-731, 2002.

[5] L. Berezansky, L. Idels, and L. Troib, "Global dynamics of Nicholson-type delay systems with applications," Nonlinear Analysis: Real World Applications, vol. 12, no. 1, pp. 436-445, 2011.

[6] L. Berezansky, E. Braverman, and L. Idels, "Nicholson's blowflies differential equations revisited: main results and open problems," Applied Mathematical Modelling, vol. 34, no. 6, pp. 1405-1417, 2010.

[7] H.-S. Ding and J. J. Nieto, "A new approach for positive almost periodic solutions to a class of Nicholson's blowflies model," Journal of Computational and Applied Mathematics, vol. 253, pp. 249-254, 2013.

[8] L. Wang, "Almost periodic solution for Nicholson's blowflies model with patch structure and linear harvesting terms," Applied Mathematical Modelling, vol. 37, no. 4, pp. 2153-2165, 2013.

[9] W. Wang, L. Wang, and W. Chen, "Existence and exponential stability of positive almost periodic solution for Nicholsontype delay systems," Nonlinear Analysis: Real World Applications, vol. 12, no. 4, pp. 1938-1949, 2011.

[10] B. Liu and S. Gong, "Permanence for Nicholson-type delay systems with nonlinear density-dependent mortality terms," Nonlinear Analysis: Real World Applications, vol. 12, no. 4, pp. 1931-1937, 2011.

[11] B. Liu, "Global exponential stability of positive periodic solutions for a delayed Nicholson's blowflies model," Journal of Mathematical Analysis and Applications, vol. 412, no. 1, pp. 212-221, 2014.

[12] P. Amster and A. Déboli, "Necessary and sufficient conditions for the existence of periodic solutions of a Nicholson type 
delay system," Differential Equations and Dynamical Systems, vol. 27, no. 4, pp. 439-447, 2019.

[13] C. Xu, P. Li, and S. Yuan, "New findings on exponential convergence of a Nicholson's blowflies model with proportional delay," vol. (2019), Article ID 358, https://advancesindifference equations.springeropen.com/.

[14] Y. Xu and Q. Cao, "Global asymptotic stability for a nonlinear density-dependent mortality Nicholsons blowflies system involving multiple pairs of time-varying delays," vol. 123, 2020 https://www.x-mol.com/paper/journal/10646? _ detail $=1241037100007493632$.

[15] C. Huang, X. Yang, and J. Cao, "Stability analysis of Nicholson's blowflies equation with two different delays," Mathematics and Computers in Simulation, vol. 171, pp. 201-206, 2020.

[16] X. Mu, D. Jiang, T. Hayat, A. Alsaedi, and B. Ahmad, "Dynamical behavior of a stochastic Nicholson's blowflies model with distributed delay and degenerate diffusion," Nonlinear Dynamics, vol. 103, no. 2, pp. 2081-2096, 2021.

[17] A. Muhammadhaji, Z. Teng, and X. Abdurahman, "Permanence and extinction analysis for a delayed ratio-dependent cooperative system with stage structure," Afrika Matematika, vol. 25, no. 4, pp. 897-909, 2014.

[18] A. Muhammadhaji and Z. Teng, "Permanence and extinction analysis for a periodic competing predator-prey system with stage structure," International Journal of Dynamics and Control, vol. 5, no. 3, pp. 858-871, 2017. 